\title{
Technology Policy Recommendations Using Artificial Intelligence
}

\author{
Ashita Anuga', Minh Nguyen', Dominick Perini' ${ }^{1}$ Andrei Svetovidov', Amanda Tolman², Qasim \\ Wani $^{1}$, Feras Batarseh ${ }^{1}$ \\ ${ }^{1}$ Virginia Tech, ${ }^{2}$ Radford University \\ ashita23@vt.edu,mnguyen0226@vt.edu,perindom@vt.edu, andreis@vt.edu, atolman@ radford.edu, qasim@vt.edu, batarseh@vt.edu
}

\begin{abstract}
Conventionally, the approach to policy making includes weighing the costs and benefits (i.e., tradeoffs) of certain choices to calculate expected outcomes. However, quantifying choices is not always straightforward without understanding many factors such as time, causal effects, and associations - making it difficult to label policy as either a failure or a success. Accordingly, our work proposes utilizing Artificial Intelligence (AI) algorithms to assess the impact of policy (state-level science and technology policies as an example). Our approach allows for an efficient policy generating process, providing policymakers with insights based on previous legislation and historical data for their respective states. Leveraging AI this way stimulates humanlike learning which can yield better results with the subjective behavior of public policy. Our approach consists of collecting datasets relevant to science and technology policies, utilizing AI to create methods for determining the best path forward, testing the validity of the algorithms using AI assurance, and measuring attributions to determine which components contribute to the outcomes most effectively. Using AI provides context relevant to the impacts of certain policies, and an overall data-driven approach that mitigates depending solely on expert's judgment, subjective experiences, or ad-hoc processes.
\end{abstract}

Copyright $($ C 2021, by the authors. All rights reserved. 